\title{
Common and Uncommon CTO Complications
}

\author{
Johannes Rigger, ${ }^{1,2}$ Colm G Hanratty ${ }^{1}$ and Simon J Walsh ${ }^{1}$ \\ 1. Cardiology Department, Belfast Health \& Social Care Trust, Belfast, Northern Ireland, UK; 2. Kantonsspital, St Gallen, Switzerland
}

\begin{abstract}
Despite the ongoing development of technical skills, increasing operator experience and improvements in medical devices, percutaneous coronary interventions (PCI) for chronic total occlusions (СТO) are still the most challenging procedures in interventional cardiology for coronary artery disease. Due to the complexity of the procedures, there is an increased complication rate compared with PCls for the treatment of non-occlusive disease. This may significantly increase procedural morbidity and potentially mortality. CTO-PCI related complications include all the usual complications that are seen in routine PCl in addition to unique issues such as inadvertent occlusion of donor vessels or injury of collateral channels causing haemodynamic instability or ischaemia. To minimise the morbidity associated with these procedures, it is important to be aware of potential complications and recognise them in a timely fashion. Should they arise, operators should be able to deal with them in a safe and efficient manner.
\end{abstract}

\section{Keywords}

Chronic total occlusions, percutaneous interventions, complications, coronary disease, stents

Disclosure: The authors have no conflicts of interest to declare.

Received: 1 May 2018 Accepted: 23 July 2018 Citation: Interventional Cardiology Review 2018;13(3):121-5. DoI: https://doi.org/10.15420/icr.2018.10.2

Correspondence: Johannes Rigger, Cardiology Department, Belfast Health \& Social Care Trust, Belfast, Northern Ireland, UK; and Kantonsspital, St Gallen, Switzerland.

E: johannes.rigger@gmail.com

Chronic total occlusions (СTO) are a common finding at angiography in patients with coronary artery disease (CAD); they are present in $\sim 20 \%$ of cases at angiography (excluding those with acute $\mathrm{Ml}$ or prior coronary artery bypass graft (CABG). ${ }^{1}$ Data from the same Canadian registry showed that only $10 \%$ of CTO patients had had a percutaneous coronary intervention (PCl) attempted to treat CTO, with only $7 \%$ successfully revascularised by this procedure. Recent data continue to demonstrate a low rate of CTO $\mathrm{PCl}$ in the UK, with approximately $5 \%$ of all $\mathrm{PCl}$ lesions being a CTO procedure and $12-13 \%$ of $\mathrm{PCl}$ for stable lesions being a $\mathrm{CTO} \mathrm{PCl}^{2}$

The presence of a CTO has historically led patients to be referred for a CABG or a recommendation for medical therapy. Rates of CTO PCI most probably continue to be low because of the perception that these lesions are technically challenging to deal with, and that the procedure has low success rates and higher complication rates. This is despite a large body of evidence demonstrating that high procedural success rates (of $~ 90 \%$ ) are now routine across large groups of patients in different geographies, as well as among operators and centres with varying experience. ${ }^{3-5}$

\section{Rates of Adverse Events Associated with CTO PCI}

Traditionally, $\mathrm{PCl}$ for non-occlusive disease is viewed as a low-risk procedure for patients with stable CAD. Rates of early Q-wave MI, emergency $\mathrm{CABG}$, cerebrovascular accident (CVA) and mortality are reported at $0.03 \%, 0.02 \%, 0.04 \%$ and $0.14 \%$, respectively, in a national audit of $>100,000 \mathrm{PCls}$ annually. ${ }^{2}$

However, as with all registries, the potential for under-reporting complications (especially events that occur several hours after the
$\mathrm{PCl}$ ) should always be borne in mind. For example. in recent large $\mathrm{PCl}$ studies, the in-hospital major adverse cardiac events (MACE) rates associated with left main $\mathrm{PCl}$ were noted to be $\sim 4 \%^{6}$ and $5 \%^{7}$ when there were systematic follow-up and clinical events committees for adjudication. This number is well in excess of the $\sim 0.2 \%$ MACE rate (death, stroke or Q-wave MI) for PCl for stable CAD and non-occlusive disease noted in the UK audit.

Rates of inpatient mortality and MACE from a single-centre series of 25 years of CTO PCl has, reassuringly, suggested success rates are increasing while adverse events are declining over time. ${ }^{8}$ Inpatient adverse events were described among 18,061 patients and 18,941 target СTO vessels in a meta-analysis. ${ }^{9}$ These data suggest low risks for mortality $(0.2 \%)$, emergent coronary bypass graft $(0.1 \%)$, stroke $(0.01 \%)$ and contrast nephropathy $(3.8 \%) .{ }^{9}$ Perforation was reported at $2.0 \%$ with cardiac tamponade in $0.3 \%$. The approach taken can also influence adverse events, with retrograde procedures being associated with higher numbers of these outcomes. ${ }^{10}$ While mortality remains low (0.7\%), collateral perforation (6.9\%) and tamponade (1.4\%) were noted amongst 3,482 patients undergoing retrograde СTO $\mathrm{PCI}$ procedures.

As discussed above, the accuracy of these data (that are almost all registry based) may be limited and, as such, they should be interpreted cautiously. The OPEN CTO registry has been reported recently. In contrast to previous reports of CTO PCl outcome, this study was linked to the National Cardiovascular Data Registry registry to ensure that all consecutive patients were assessed. Furthermore, angiographic core laboratory and central clinical event adjudication were applied for all patients. Under these circumstances of systematic and robust clinical follow-up for contemporary СTO $\mathrm{PCl}$, adverse event rates were noted to be more frequent. ${ }^{5}$ In-hospital mortality was found 
Table 1: Summary of In-Hospital Complications in Patients Undergoing $\mathrm{PCl}$ in Non-CTO and CTO Vessels

\begin{tabular}{lll} 
In-Hospital Complications & PCI & CTO PCI \\
Q-wave myocardial infarction & $0.02 \%$ & $2.5 \%$ \\
\hline Emergency CABG & $0.03 \%$ & $0.1 \%$ \\
\hline Stroke & $0.04 \%$ & $0.01 \%$ \\
\hline Mortality & $0.14 \%$ & $0.2-0.9 \%$ \\
\hline Perforation & $0.38 \%$ & $2-4.8 \%$ \\
\hline
\end{tabular}

Source: Ludman and British Cardiovascular Intervention Society, 2017;' Prasad et al., 2007;" Patel et al., 2013, ${ }^{10}$, El Sabbagh et al., 2014"

to be $0.9 \%$ and a coronary perforation requiring intervention was $4.8 \%$. Modern CTO PCl techniques do afford intervention for much more complex disease and this is likely to be the main factor in an upturn in complications. Nevertheless, these data should be viewed as being derived from a gold standard for outcome reporting. Where outcome data are reported without this level of robust follow-up, discernment with regards to their absolute validity is required.

Table 1 gives a summary of in-hospital complications in patients undergoing $\mathrm{PCl}$ in non-CTO and CTO vessels.

Regardless of the small absolute numbers of serious complications, all operators should be aware of these events and should be able to treat them immediately if needed. CTO-related complications can be considered as acute or late as well as cardiac (coronary and noncoronary) or extracardiac complications (Figure 1). ${ }^{11}$

Specific risks in CTO $\mathrm{PCl}$ include: acute myocardial infarction due to compromise of collateral flow; coronary perforation with pericardial blood extravasation with or without cardiac tamponade; damage of the proximal occluded segment due to deep guide catheter intubation; donor vessel damage (mechanical or thrombotic) during retrograde crossing; and renal failure due to excessive contrast load. ${ }^{11}$

\section{Perforation and Collateral Channel Injury}

Coronary perforation is a well-known complication of CTO PCI. Left undiagnosed or untreated, this may become life threatening. The commonest scenario leading to perforation is of a guide wire exiting from the CTO body during attempted antegrade wire escalation (AWE). As long as the wire is not followed by secondary equipment, this is typically a relatively forgiving scenario without significant clinical sequelae.

Perforations leading to significant bleeding are unusual, but those requiring an intervention ( $1-2 \%$ in registries and almost $5 \%$ in OPEN $\mathrm{CTO}^{5}$ ) are common enough to require familiarity with corrective manoeuvres. In the authors' experience, those associated with tamponade are most commonly due to collateral channel injury during a retrograde procedure or from unrecognised distal wire exits after antegrade crossing. Other potential causes include passing equipment out of small branches because the operator does not realise they are not in the main vessel architecture, or where stent deployment (or postdilation) leads to contrast extravasation as would also be seen in routine $\mathrm{PCl}$ (Figure 2)

Should a significant perforation occur, the first step is to prevent continuing blood loss. This is usually achieved by inflating a balloon (1:1 sizing) in the proximal portion of the leaking vessel. If there is a significant bleed while working with a $6 \mathrm{~F}$ or $7 \mathrm{~F}$ guide catheter, it is often useful to site a second guide catheter in the same vessel (a "ping-pong guide"). This will allow the operator to maintain control of the bleed, while briefly deflating the occlusion balloon, then reinflating it once equipment has passed distally to facilitate distal intervention. However, a ping-pong guide is not required if a $8 \mathrm{~F}$ guide is used when covered stent delivery and continued balloon inflation can be managed via a single guide. Operators should be aware that perforations from epicardial collaterals can be "fed" from multiple sources and that control of continuing bleeding can be challenging.

The next question is whether there is haemodynamic compromise. If the patient has hypotension, an echocardiogram can confirm a diagnosis of cardiac tamponade and aid subsequent pericardiocentesis. With this in mind, it is important that catheter laboratories that perform regular CTO $\mathrm{PCl}$ have this equipment to hand with immediate availability in case it is needed for emergent use.

Subsequently, the operator needs to identify the exact source of bleeding and the steps that are most appropriate to prevent ongoing blood loss. A specific inventory is necessary to be able to resolve the underlying abnormality. Potential solutions include the injection of autologous fat through a microcatheter (often effective for distal branch wire exits), coils (frequently required for collateral channel perforation), micro-beads, thrombin or covered stents. An appropriate range of this equipment must be available at all СТO PCI centres.

Some specific scenarios can arise with collateral channel injury during retrograde approaches that require recognition and different steps for resolution. When there has been exit into a cardiac chamber, these events are almost always benign and self-limiting. It is important that the nature of any ongoing extravasation is understood. Fat, thrombin or micro-beads should never be injected if there is a collateral leak into a left heart chamber as these can potentially embolise and cause a stroke. very large septal haematomas have also been described, including those that lead to a "dry tamponade". Occasionally, these will spontaneously decompress ${ }^{12}$ by forming a self-limiting ventricular septal defect. Where a very large septal haematoma leads to patient compromise, this can be decompressed by percutaneous intervention. Under these circumstances, if the operator can pass a wire from the left coronary side via the haematoma cavity to the right ventricle, passage of a micro-catheter or $2 \mathrm{~mm}$ balloon to dilate this connection may allow the haematoma to drain without further compromise to the patient.

Other potential sequelae of collateral channel exit are localised pressure effects, which are seen most often in the patients who have had a CABG. It is not unusual to see right ventricle free wall haematomas form when there has been over-aggressive dissection in the right coronary artery or when there has been dissection into branches with or without equipment exit in a "closed-chest" patient. Typically, supportive measures with intravenous fluids and/or inotropes will be sufficient to support the patient and allow spontaneous resolution. In the post-CABG setting, leaks from atrioventricular groove epicardial collaterals can lead to a left atrial pseudo myxoma and impaired mitral inflow..$^{13}$ These are often best diagnosed by CT angiography and may require localised drainage using CT guidance ${ }^{13}$ or open surgery in rare circumstances.

Where retrograde approaches are considered, interventional 


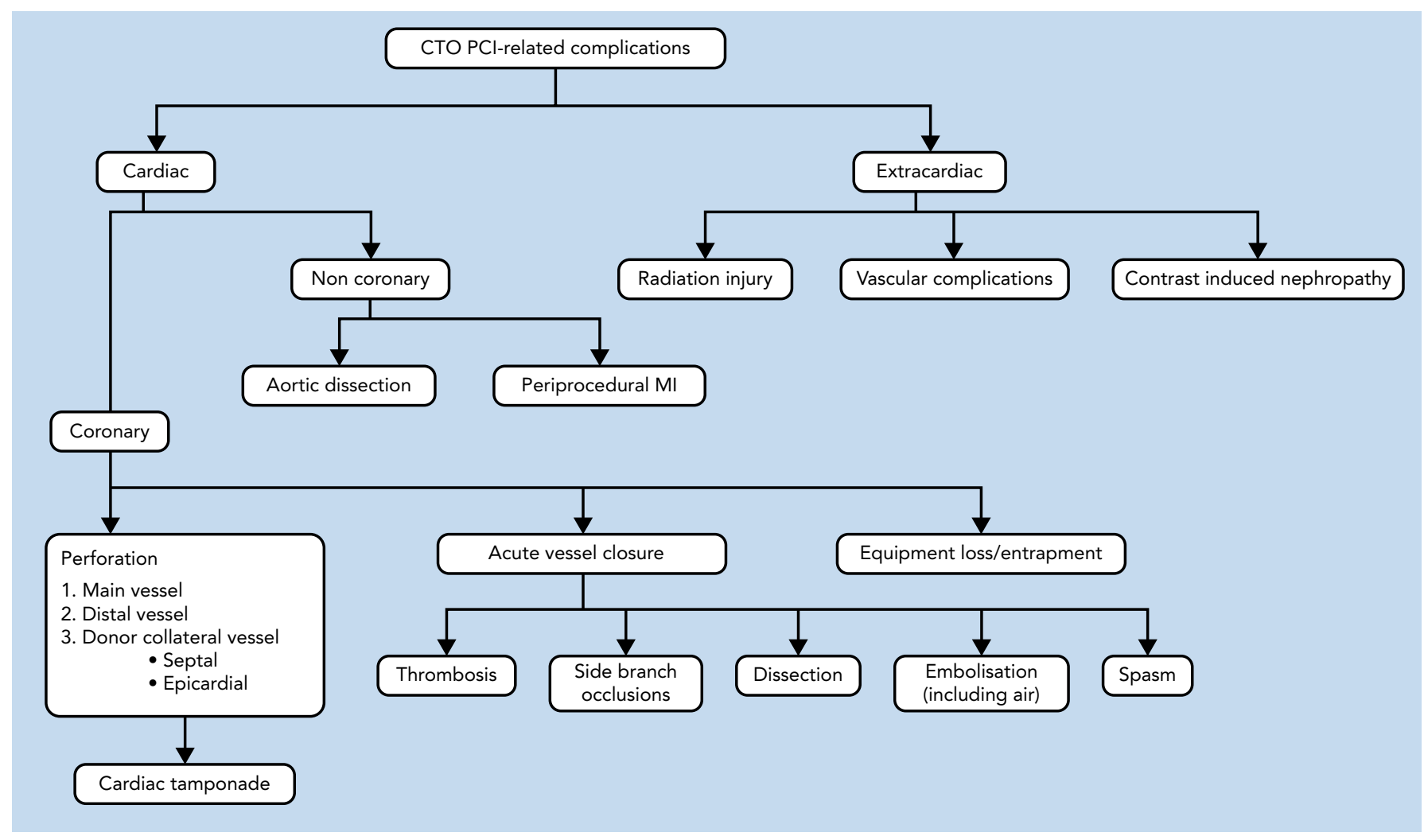

Source: El Sabbagh et al (2014)

cardiologists should be mindful of the hierarchical risk associated with each approach. In general, the use of patent (but diseased) vein grafts is safe. Septal collaterals are usually forgiving, and minor perforations tend to be well tolerated. Epicardial channels are less forgiving should a complication occur and should be considered as the last option. The authors would advocate avoiding the use of a left internal mammary artery (especially as a last remaining conduit) where at all possible.

Finally, cardiac surgery may be required as a bailout option for percutaneously unresolvable bleeding. Centres that do not have on-site cardiac surgery facilities should strongly consider whether the use of epicardial collaterals and certain techniques is appropriate. In addition, clear pathways should be established for the transfer of patients to surgical centres under emergent circumstances should the need arise.

\section{Сто Strategies, Vascular Access, Radiation Injury and Contrast Nephropathy}

A number of strategies can be adopted for CTO PCl. Many lesions are amenable to two or more approaches. An algorithmic approach to CTO $\mathrm{PCl}$ was first suggested in 2012; this recommends using the anatomy of the lesion to guide the initial strategy to approach the lesion. ${ }^{14}$ Four key questions guide the operator towards AWE, antegrade dissection and re-entry (ADR), retrograde wire escalation (RWE) or retrograde dissection and re-entry (RDR). An important aspect of a hybrid approach to $\mathrm{CTO} \mathrm{PCl}$ is that operators must be able to recognise when they are entering a failure mode and efficiently switch between approaches. The main goal of this strategic switching is to minimise procedure duration, radiation exposure and contrast load. The use of the hybrid approach has been shown to be associated with high procedural success rates ( $\sim 90 \%$ per lesion) with acceptable use of contrast and radiation. ${ }^{3-5,15,16}$
Figure 2: Type III Perforation

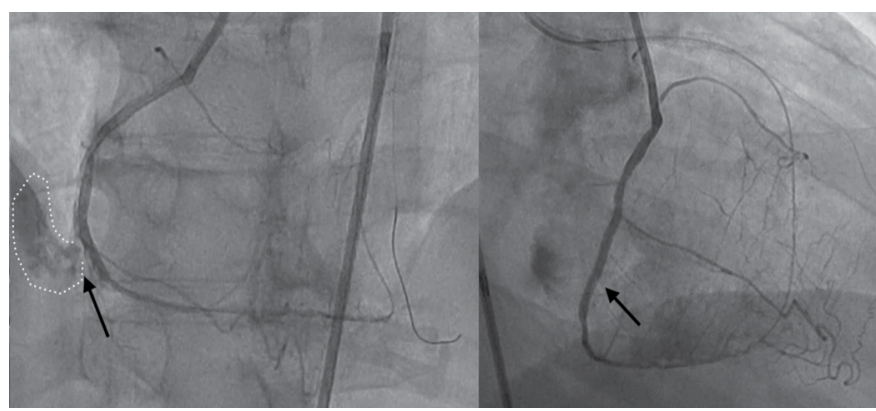

(a) Angiography showing a type III perforation (black arrow) after stenting of the right coronary artery after CTO revascularisation; (b) after deployment of two covered stents, with complete sealing of the right coronary artery perforation.

Several basic steps can be taken to reduce radiation doses. These include reducing frame rates for both fluoroscopy and acquisition runs, minimising the use of acquisition runs to points in the case where they are both necessary and useful, and strongly encouraging operators to be vigilant around the use of X-rays. Where CTO PCIs are performed in institutions with multiple $\mathrm{x}$-ray systems, performing these procedures with the most modern $\mathrm{x}$-ray equipment is likely to reduce exposure. ${ }^{17}$

Contrast nephropathy should be considered as a potential adverse event related to CTO PCl. Minimising contrast use is key and, once again, efficient and appropriate use of СTO strategies is pertinent to prevent wasting contrast. However, several peri-procedural manoeuvres can reduce this risk. These include stopping nephrotoxic agents for 48 hours before the procedure (especially angiotensinconverting-enzyme [ACE] inhibitors or angiotensin II receptor blockers 
[ARBs]), pre-hydrating patients with intravenous fluids and/or the use of the RenalGuard system.

Historically, the use of certain strategies within PCl led to consideration of the use of $8 \mathrm{~F}$ guiding systems (especially for ADR). Data have shown an association between sheath size and an increased risk for major bleeding ${ }^{19}$. Fortunately, the progressive evolution of CTO PCl dedicated technologies means that 8 F-guiding systems are rarely necessary now. Vascular complications related to femoral arterial access still occur in a significant number of cases in contemporary practice $(0.3 \%$ of patients required treatment in OPEN CTO, with haematomas noted in $4.3 \%$; major vascular complications were noted in $2.5 \%$ of RECHARGE patients ${ }^{4}$ ). The routine use of fluoroscopy to guide femoral puncture ${ }^{18}$ and/or the use of ultrasound may help to minimise adverse events. However, bilateral transradial access will obviate the risk of any femoral bleeding and is now feasible for the vast majority of CTO cases; it should be considered as an option where feasible and indicated.

\section{Donor Vessel Issues}

In general, if there is no retrograde approach there are limited implications from seating a guide catheter in the non-CTO (contralateral) vessel. Rarely, and often in the context of profound ischaemia with very severe left ventricular dysfunction, bilateral contrast injection can lead to ischaemia or arrhythmia. Under such circumstances, sequential single catheter injections may be safer.

Retrograde approaches raise numerous and specific issues related to the donor vessel. Any compromise to this system is potentially catastrophic, as the territory supplied is at risk as well as the СтO territory. Complications can lead to extensive ischaemia and rapid haemodynamic collapse and the operator should always consider this possibility if a patient becomes acutely unwell.

Donor-vessel occlusion due to thrombus formation should be avoided at all costs. While this complication can occur in the target vessel during antegrade CTO PCl, the risk is higher for retrograde access. The excess of intracoronary equipment is associated with stasis in the guide catheters. Therefore, meticulous attention must be paid to optimal anticoagulation using the activated clotting time (ACT) to minimise the risk for donor system thrombosis. After an initial bolus of $100 \mathrm{U} / \mathrm{kg}$, the ACT should be monitored to maintain a level $>300$ seconds (some advocate 350 seconds) to minimise the risk. Back-bleeding of guide catheters and recurrent flushing is also recommended after material exchange.

Donor vessel dissection causing occlusion is relatively rare. This complication is most likely to occur as retrograde equipment is withdrawn from the СТO vessel. Under these circumstances, friction and energy that has been stored in the system during micro-catheter crossing is released. An equal and opposite reaction can occur as equipment is brought out of the CTO territory back to the donor guide and the guiding catheter can be sucked into the donor vessel. This is best avoided by withdrawing the donor guide catheter well away from the ostium of the artery as the equipment is being withdrawn, and continuously imaging to ensure that the catheter is not pulled deeply into the donor vessel. The authors would also advocate the simple step of leaving a workhorse guide wire in the distal segment of the donor artery throughout the procedure to facilitate bail-out intervention should a problem arise.
Occasionally, retrograde approaches can lead to kinking of the donor vessel, especially when the micro-catheter is advanced into a tortuous native vessel with significant angulation into the donor collateral. Under these circumstances, options are limited. If ischaemia and compromise occur, a braided micro catheter (Finecross, Caravel etc) that is more flexible and has a lower profile than a coil-based micro-catheter (Corsair, Turnpike etc) may afford continued retrograde manoeuvres. However, if these devices are also associated with compromise of the donor vessel, then the retrograde approach may have to be altered (to an alternative collateral) or abandoned.

Equipment loss and entrapment are rare. Stents can come loose from their delivery balloon during attempted delivery through long, calcific and tortuous lesions. Low-threshold use of guide catheter extensions seems to obviate this risk in the majority of CTO PCI. Should this issue occur, retrieval is the same as during routine $\mathrm{PCl}$.

Specific to retrograde CTO PCl, guide wires can become knotted after crossing into the CTO territory though this is rare and avoidable. This is a risk where knuckling has been performed, especially if the operator mistakenly "spins" the knuckle wire (this should be avoided). Pushing the retrograde equipment is more likely to free it up than pulling. Swapping the micro-catheter with a retrograde balloon and inflating this at the problem area may loosen any tissue that is entangled with the equipment. Similarly, siting antegrade equipment in the same area and ballooning from this direction may also be helpful.

\section{Myocardial Injury Associated with CTO PCI}

It is not uncommon to see small increases in cardiac enzymes associated with retrograde approaches. This occurs due to the ischaemia that is invoked by collateral channel occlusion. Usually, this is well tolerated by the patient and not associated with ischaemia on the ECG. A low-level troponin elevation is of questionable clinical relevance. However, if a dominant collateral channel is used and the patient experiences significant cardiac ischaemia and chest pain when this is crossed, the operator should consider an alternative collateral or strategy to prevent significant myocardial infarction.

During ADR or RDR procedures, it is imperative that attention is paid to large side branches. These should never be sacrificed by the strategy chosen and, where the loss of a significant vessel is possible or likely, an alternative approach should be taken.

\section{When to Stop Revascularisation}

It is crucial that the treating physician is capable of recognising futility during the procedure. A contrast (depending on baseline renal function) and radiation limit (many would advocate 4 Gy) should be set and, if no meaningful progress has been made, the procedure should be discontinued. Where a CTO PCl attempt had been made with some progress, dilating the proximal cap and modifying the CTO segment by balloon dilation (an "investment procedure") can alter the anatomy and facilitate another procedure after a few weeks. Under these circumstances, repeat attempts are associated with very high success rates ( $90 \%) .^{3}$

\section{Conclusions}

The tremendous improvement of equipment in coronary intervention allows practitioners to revascularise a broad range of CTO lesions in contemporary practice. Many of the most complex lesions will require the use of advanced techniques. CTO interventions can 
be performed safely in centres willing to make the commitment to training and education to achieve a high level of success for these challenging procedures. A significant part of modern CTO PCl includes an awareness of the potential pitfalls of the procedure, being able to recognise these events promptly when they arise and the ability to resolve them when they do occur. It is important that operators are suitably trained and centres are appropriately equipped for this.
1. Fefer P, Knudston ML, Cheema AN, et al. Current perspectives on coronary chronic total occlusions. The Canadian Multicenter Chronic Total Occlusions Registry. I Am Coll Cardio Multicenter Chronic Total Occlusions Registry. J Am Coll Cardior 2012;59:991-7.

2. Ludman P, British Cardiovascular Intervention Society. BCIS audit returns. Adult interventional procedures January 2016 to December 2016. Available at Available at: www.bcis.org.uk/ resources/audit-results/ (accessed 23 August 2018)

3. Wilson WM, Walsh SJ, Yan AT, et al. Hybrid approach improves success of chronic total occlusion angioplasty. Heart 2016;102:1486-93. https://doi.org/10.1136/ heartjinl-2015-308891. PMID:27164918.

4. Maeremans J, Walsh $\mathrm{S}$, Knaapen $\mathrm{P}$, et al. The hybrid algorithm for treating chronic total occlusions in Europe: the RECHARGE registry. J Am Coll Cardiol 2016;68:1958-70. https://doi. org/10.1016/j.jacc.2016.08.034. PMID:27788851.

5. Sapontis J, Salisbury AC, Yeh RW, et al. Early procedural and health status outcomes after chronic total occlusion angioplasty. A report from the OPEN-CTO Registry (Outcomes, Patient Health Status, and Efficiency in Chronic Total Occlusion Hybrid Procedures). J Am Coll Cardiol Inty 2017;10:1523-34. https://doi.org/10.1016/j.jcin.2017.05.065. PMID:28797429.

6. Mäkikallio T, Holm NR, Lindsay M, et al. Percutaneous coronary angioplasty versus coronary artery bypass grafting in treatment of unprotected left main stenosis (NOBLE): a prospective, randomised, open-label, non-inferiority trial. Lancet 2016;388:2743-52. https://doi.org/10.1016/S01406736(16)32052-9. PMID:27810312.

7. Stone GW, Sabik JF, Serruys PW, et al for the EXCEL Tria Investigators. Everolimus-eluting stents or bypass surgery for left main coronary artery disease. N Engl J Med 2016;375:222335. https://doi.org/10.1056/NEIM001610227. PMID:27797291.

8. Prasad A, Rihal CS, Lennon RJ. Trends in outcomes after percutaneous coronary intervention for chronic total occlusions. A 25-year experience from the Mayo Clinic J Am Coll Cardiol 2007;49:1611-8. https://doi.org/10.1016/ j.jacc.2006.12.040. PMID:17433951.

9. Patel VG, Brayton KM, Tamayo A. Angiographic success and procedural complications in patients undergoing percutaneous coronary chronic total occlusion interventions: a weighted meta-analysis of 18,061 patients from 65 studies. JACC Cardiovasc Interv 2013:6:128-136. https://doi.org/10.1016/ j.jcin.2012.10.011. PMID:23352817

10. El Sabbagh A Patel VG, Jeroudi OM. Angiographic success and procedural complications in patients undergoing retrograde percutaneous coronary chronic total occlusion interventions: a weighted meta-analysis of 3,482 patients from 26 studies. Int J Cardiol 2014;174: 243-8. https://doi. org/10.1016/j.ijcard. 2014.04.004. PMID:24768461.

11. Patel Y, Depta JP, DeMartini TJ. Complications of chronic total occlusion percutaneous coronary intervention. Interv Cardiol 2013;5:567-75. https://doi.org/10.2217/ica.13.48.

12. Fairley SL, Donnelly PM, Hanratty CG, Walsh SJ. Images in cardiovascular medicine. Interventricular septal hematoma and ventricular septal defect after retrograde intervention for a chronic total occlusion of a left anterior descending for a chronic total occlusion of a left anterior descending coronary artery. Circulation 2010;122(20):e518-21. https://doi.
org/10.1161/CIRCULATIONAHA.110.976555. PMID:21098463.

13. Wilson WM, Spratt JC, Lombardi WL. Cardiovascular collapse post chronic total occlusion percutaneous coronary intervention due to a compressive left atrial hematom managed with percutaneous drainage. Catheter Cardiovasc
Interv 2015;86(3):407-11. https://doi.org/10.1002/ccd.25571. PMID:24909556.

14. Brilakis ES, Grantham JA, Rinfret $S$, et al. A percutaneous treatment algorithm for crossing coronary chronic total occlusions. JACC Cardiovasc Interv 2012;5:367-79. https://doi.org/10.1016/j.jcin.2012.02.006. PMID:22516392.

15. Pershad A, Eddin M, Girotra S, et al. Validation and incremental value of the hybrid algorithm for CTO PCl. Catheter Cardiovasc Interv 2014;84:654-9. https://doi.org/10.1002/ ccd.25370. PMID:24403122

16. Christopoulos G, Karmpaliotis D, Alaswad K, et al. Application and outcomes of a hybrid approach to chronic total occlusion percutaneous coronary intervention in a contemporary multicenter US registry. Int I Cardiol 2015;198:222-8. https://doi.org/10.1016/j.ijcard.2015.06.093. PMID:26189193; PMCID.PMC4554818.

17. MCNeice A, Brooks M, Hanratty CG, et al. A retrospective study of radiation dose measurements comparing different cath lab X-ray systems in a sample population of patients undergoing percutaneous coronary intervention for chronic total occlusions. Catheter Cardiovasc Interv 2018; Feb 7. https://doi.org/10.1002/ccd.27541. PMID: 29411518. Epub ahead of print.

18. Fairley SL, Lucking AJ, MCEntegart M, et al. Routine use of fluoroscopic-guided femoral arterial puncture to minimise vascular complication rates in CTO intervention: multicentre UK experience. Heart Lung Circ 2016;25(12):1203-9. https://doi.org/10.1016/j.hlc.2016.04.006. PMID:27265645.

19. Doyle BJ, Ting HH, Bel MR, et al. Major femoral bleeding complications after percutaneous coronary intervention. JACC Cardiovasc Interv 2008;2:202-9. https://doi.org/10.1016/ j.cin.2007.12.006. PMID:19463301. 